\title{
Racial disparities in advanced stage colorectal cancer survival
}

\author{
Kristin Wallace ${ }^{1}$, Elizabeth G. Hill' ${ }^{1}$, David N. Lewin ${ }^{2}$, Grace Williamson ${ }^{1}$, Stephanie \\ Oppenheimer $^{1}$, Marvella E. Ford ${ }^{1}$, Michael J. Wargovich ${ }^{3}$, Franklin G. Berger ${ }^{4}$, Susan W. \\ Bolick $^{5}$, Melanie B. Thomas ${ }^{6}$, and Anthony J. Alberg ${ }^{1}$ \\ ${ }^{1}$ Division of Epidemiology and Biostatistics, Department of Medicine, Medical University of South \\ Carolina, Charleston, SC \\ ${ }^{2}$ Department of Pathology, Medical University of South Carolina, Charleston, SC \\ ${ }^{3}$ Department of Pharmacology, Medical University of South Carolina, Charleston, SC \\ ${ }^{4}$ Center for Colon Cancer Research, Department of Biological Sciences, University of South \\ Carolina, Columbia, SC \\ ${ }^{5}$ South Carolina Central Cancer Registry, Columbia, SC \\ ${ }^{6}$ Division of Medical Oncology, Department of Medicine, Medical University of South Carolina, \\ Charleston, SC
}

\begin{abstract}
Purpose-African Americans (AA) have a higher incidence and lower survival from colorectal cancer (CRC) compared to European Americans (EA). In the present study, statewide, populationbased data from South Carolina Central Cancer Registry (SCCCR) is used to investigate the relationship between race and age on advanced stage CRC survival.
\end{abstract}

Methods-The study population was comprised of 3865 advanced pathologically documented colon and rectal adenocarcinoma cases diagnosed between 01 January 1996 and 31 December 2006: 2673 (69\%) EA and 1192 (31\%) AA. Kaplan-Meier methods were used to generate median survival time and corresponding $95 \%$ confidence intervals (CI) by race, age, and gender. Factors associated with survival were evaluated by fitting Cox proportional hazards $(\mathrm{CPH})$ regression models to generate Hazard Ratios (HR) and 95\% CI.

\begin{abstract}
Results-We observed a significant interaction between race and age on CRC survival ( $\mathrm{p}=$ 0.04). Among younger patients (<50 years), AA race was associated with a 1.34 ( $95 \%$ CI 1.06-1.71) higher risk of death compared to EA. Among older patients, we observed a modest increase risk of death among AA men compared to EA (HR 1.16 (95\% CI 1.01-1.32) but no difference by race among women (HR 0.94 (95\% CI 0.82-1.08)). Moreover, we observed that the disparity in survival has worsened over the past 15 years.
\end{abstract}

Corresponding Author: Kristin Wallace, PhD Assistant Professor of Epidemiology Department of Medicine Medical University of South Carolina Charleston, SC Telephone: 843-876-2432 Fax: 843-876-2344 wallack@ musc.edu.

Conflict of Interest: The authors declare that they have no conflict of interest. 
Conclusions-Future studies that integrate clinical, molecular, and treatment-related data are needed for advancing understanding of the racial disparity in CRC survival, especially for those < 50 years old.

\section{Keywords}

African American; survival; metastatic; colon cancer; young-onset

\section{Introduction}

Colorectal cancer (CRC) is the third most common malignancy in the US and the second leading cause of cancer death [1]. Compared to European Americans (EA), African Americans (AA) have a 20\% higher CRC incidence rate and a 45\% higher mortality rate [1]. At least some of the excess mortality in AA is caused by the reduced survival associated with a higher prevalence of metastatic disease at diagnosis [2-4]. However, the disparity persists at each stage and is likely to be influenced by differences in treatment utilization and/or CRC tumor biology.

CRC survival has improved significantly over the past fifteen years due to the introduction of several new chemotherapeutic agents; yet, the racial difference in survival has worsened, especially among advanced stage patients [5,6]. Until the year 2000, there were few treatment options other than 5-Fluorouracil (5-FU) and leuvocourin for metastatic CRC; however, with the introduction of combination chemotherapy in 2000 (i.e., irinotecan or oxaliplatin with 5-FU) and the addition of biologic agents to the combination chemotherapy in 2004 (i.e., bevacizumab or cetuximab), the median survival has increased from 8-12 months in the mid-1990's to 18-24 months in 2008 [7]. Although few studies have examined the effect of the new therapies on CRC survival at the population level, historical evidence suggests that AA may not experience the same survival benefit as EA [5, 8, 9]. To explain, AA have been reported to have lower rates of initiation of standard therapy [10-12] and refuse therapy at a higher rate than EA [13, 14]. More recently, AA have been found to have a lower response to the newer treatment regimens, which may translate to differences in survival by race $[8,15]$.

Differences in CRC tumor biology by race, age, and or gender may influence the difference in stage-specific survival. AA compared to EA are more likely to present at diagnosis at a younger age and with features indicative of greater tumor aggressiveness, including a higher proportion of proximal compared to distal neoplasia (11-17). Proximal colonic location is associated with worse survival [16-19], especially among advanced stage CRC patients. Earlier age of onset, irrespective of race, is associated with aggressive tumor histopathology, a higher grade and stage of CRC, and a higher proportion of hereditary CRC [20-27]. Furthermore, gender may also be associated with difference in tumor aggressiveness since women appear to have a tendency to develop tumors with lower rates of metastasis and better prognosis $[28,29]$. However, few studies have considered the intersection of race, age and gender on survival.

In the present investigation, statewide, population-based data from South Carolina Central Cancer Registry (SCCCR) is used to examine if there are racial differences in survival in 
advanced stage CRC. This study is restricted to a single stage because the magnitude of the racial disparity in survival has been shown to differ by stage. This study focuses on metastatic disease because of similar recommended standard therapy, large racial difference in the relative survival, and a documented change in chemotherapy usage in both 2000 and 2004. We investigated the relationship between race and age on CRC survival during three calendar periods: (1) 1996-1999; (2) 2000-2003; and (3) 2004-2006, each corresponding to a change in chemotherapeutic regimen. We also explored the influence of clinicopathologic features by race in younger and older patients, and in males and females, and their impact on survival.

\section{Materials and methods}

\section{Study population}

The study population was comprised of 3865 advanced pathologically documented colon and rectal adenocarcinoma cases in South Carolina diagnosed between 01 January 1996 and 31 December 2006: 2673 (69\%) EA and 1192 (31\%) AA. For analyses of time-to-death, 402 subjects - 267 (66\%) EA subjects and 135 (34\%) AA subjects --could not be included because no survival time data were available, resulting in 3463 subjects available for survival analyses.

Patient cancer stage was coded using SEER staging criteria: local, regional, or distant. The present study was limited to patients who presented with distant disease (i.e. distant lymph nodes or distant sites); distant disease is equivalent to TNM stage IV. The SCCCR is a population-based, mandated data system that collects cancer incidence in South Carolina. Information on cancer mortality (deaths) is collected by the Division of Vital Registry and published by the Division of Biostatistics and Division of Public Health Informatics within the Department of Health and Environmental Control. For every case, data were collected on age, race (white, black, Hispanic, Asian, or other), gender, first-line chemotherapy treatment (yes or no), and year of diagnosis and death. Our analysis is restricted to EA and AA due to the low number of patients from other racial/ethnic groups.

\section{Clinicopathologic characteristics}

The clinicopathologic characteristics for each primary tumor include location, tumor grade, and histological type. The primary tumor was characterized according to the location within the colorectum: cecum, ascending colon, appendix, hepatic flexure, transverse colon, splentic flexure, descending colon, sigmoid colon, recto-sigmoid, or rectum. The tumor was grouped as proximal (cecum, ascending colon, appendix, hepatic flexure transverse colon), distal (splentic flexure, descending colon, sigmoid colon) or rectal (recto-sigmoid, or rectum).

Additionally, tumor grade was classified as well-differentiated, moderately differentiated, poorly differentiated, or undifferentiated. For the main analyses, tumor grade was dichotomized into low grade (well differentiated, moderately differentiated) and high grade (poorly differentiated, undifferentiated). Histological type was classified as adenocarcinoma not otherwise specified, mucinous adenocarcinoma, or signet ring cell adenocarcinoma. 


\section{Statistical Considerations}

Survival time was calculated as the time from diagnosis with distant stage CRC to death from any cause from 1 January 1996 through 31 December 2007; subjects alive as of this date were censored at the end of follow-up. Kaplan-Meier methods were used to generate median survival time and corresponding $95 \%$ confidence intervals by race, age, and gender for subjects diagnosed in three time periods: (1) before 31 December 1999, (2). between 01 January 2000 and 31 December 2003 (inclusive), and (3) on or after 01 January 2004, time intervals that were selected to correspond to changes in recommended systematic chemotherapy regimens.

Factors associated with survival were evaluated by fitting Cox proportional hazards (CPH) regression models. First, we explored the possibility of interaction between race and age by fitting separate $\mathrm{CPH}$ models for each age group in five-year increments, with race as the independent variable. We then examined smoothed scatter plots of the resulting estimated $\log$ cumulative hazards by age separately for AA and EA, and detected a highly non-linear effect. Graphically, the log cumulative hazards were relatively flat for ages under 50 years, but then increased linearly for increasing age thereafter (figure not shown). A CPH multivariable model fit using a three-knot restricted cubic spline transformation of age indicated significant nonlinearity $(\mathrm{p}=0.01)$. Furthermore, there was evidence of significant interaction between age (modeled non-linearly), race and gender $(p=0.04)$. Therefore, we performed stratified analyses for patients both under and over 50 years of age to aid in interpretability of results.

We subsequently fit separate $\mathrm{CPH}$ regression models for younger patients (under the age of 50 years) and older patients (50 years of age and older). For each model, we included main effects for location, grade, histologic type, age (continuous and linear), race, gender, firstline chemotherapy initiation, and diagnosis year. Additionally, for each model, we examined twoway interactions between: 1) race and each of following: gender, diagnosis year, location, grade, histology; and 2) gender and each of following: diagnosis year, location, grade, and histology. Interactions were retained in the final model if they were significant at level 0.05 .

\section{Results}

\section{Under age 50}

In the univariate analysis (Table 1), AA compared to EA were significantly more likely to be female and have proximal tumors. The prevalence of proximal tumors among African American men and women was $40 \%$ and $44 \%$ respectively, whereas the prevalence in European American men and women was $26 \%$ and $33 \%$ respectively. No differences were observed by race for tumor grade, histologic type, first-line chemotherapy, or year of diagnosis.

For the patients in our sample, the median follow up time was 15 months (range $=1$ to 141 months). Median survival improved significantly between 1996 and 2007 for both AA and EA but the improvement in survival differed by race (Table 2). The gain in median survival months was 262\% greater in EA compared to AA men and 200\% greater in EA women 
compared to AA women. During the study period, a growing racial disparity was evident among the younger aged-patients, especially among women. Prior to the year 2000, there was 6.5 month difference in survival between AA and EA women which had widened to an 11 month difference by the end of the follow-up period. Among men, however, the racial disparity was only evident after 2003. AA men had a 2 month survival advantage in time frame before 2000 which had changed to an 11 month lower survival by the end of followup.

In multivariable Cox proportional hazard analyses, overall survival was significantly poorer among African Americans $(\mathrm{p}=0.02)$, high tumor grade $(\mathrm{p}=0.001)$, adenocarcinoma histological type $(\mathrm{p}=0.04)$, and pre-2000 year of diagnosis ( $\mathrm{p}=0.01)$ (Table 3$)$. In addition to these main effects, an interaction was identified between gender and tumor location $(\mathrm{p}=0.01)$ whereby among women proximal location was associated with a 45\% (95\% CI 3\%-105\%) increased risk of death compared to those with distal tumors but in contrast among men, both proximal and rectal tumor location were associated with better survival compared to distal location.

\section{Over age 50}

In the univariate analysis (Table 1), AA compared to EA were significantly more likely to have proximal tumors $(\mathrm{p}=0.023)$ and have a lower tumor grade $(\mathrm{p}=0.01)$. There were no differences in proximal location between AA (48\%) and EA (51\%) women, but both groups had a higher prevalence of proximal tumors compared to those less than 50 years of age. Among AA men, however, the prevalence of proximal carcinomas at diagnosis was $45 \%$ and only $35 \%$ for EA men. No differences were observed by race for gender, histologic type, first-line chemotherapy initiation, or year of diagnosis.

For older patients, the median follow up time was 12 months (range $=1$ to 143 months). Median survival improved modestly from 1996 through the end of follow-up (Table 2). Overall, median survival improved for all patient groups. EA men had a $20 \%$ greater improvement in survival compared to AA men yet EA and AA women had equivalent improvements. During the study period, a marginal racial disparity emerged recently among men. Prior to the year 2003, there was no significant racial disparity among patients 50 years or older but by the end of follow-up there was a statistically significant difference in survival $(\mathrm{p}<0.02)$.

In multivariable Cox proportional hazard analyses, factors significantly associated with worse survival were older age ( $\mathrm{p}<0.0001)$, not initiating first-line chemotherapy $(\mathrm{p}<$ 00001), higher tumor grade ( $\mathrm{p}<0.0001)$, proximal tumor location $(\mathrm{p}<0.0001)$, rectal location ( $\mathrm{p}=0.041)$, and diagnosis pre-2000 ( $\mathrm{p}=0.02)$. Furthermore, there was an interaction between gender and race $(\mathrm{p}=0.04)$ on overall survival (Table 3$)$, which was based on the observation that among men, AA were $16 \%$ (95\% CI 1\%-32\%) more likely to die compared to EA yet no difference was observed among women. 


\section{Discussion}

In this analysis of South Carolina Central Cancer Registry (SCCCR) data, key findings were that AA had worse overall survival for advanced stage colorectal cancer. This racial disparity was largely concentrated among CRC patients under 50 years of age. A disconcerting observation was that the racial disparity has worsened during the past 15 years. In the survival analysis, we observed that among the younger patients, AA had worse survival especially AA females with proximal tumors. For older patients, AA males had worse survival, but the association with race was not nearly as pronounced as it was in the younger age groups.

Despite improvements in survival among those with advanced stage CRC, our study highlights the high fatality rate of this disease, and thus emphasizing the importance of prevention and early detection of CRC. In the present study, there were a higher percentage of stage IV CRC cases under the age of 50 years in AA than EA (17\% vs. 12\%) and the prognosis of AA patients was worse suggesting a more aggressive phenotype. This touches on the broader issue of developing strategies for identifying high-risk patients who have not yet reached the age for recommended screening. These findings are thus in line with the recommendations of two national organizations that recommend AA begin CRC screening earlier than EA, 40 [30] or 45 [31] years of age.

Our findings of poorer CRC survival in AA compared to EA parallel those of several previous investigations. Our finding that the disparity among late stage CRC patients is largely concentrated among the younger patients is novel. In general, the racial disparity has been shown to occur at each stage for both men and women and has worsened during the past 40 years (23). Most (10, 24-34), but not all (35-37), studies have observed that the disparity persists even after adjustment for variables such as age, sex, stage, socioeconomic status, insurance status, and in some studies pathologic characteristics. After accounting for these factors, the survival differences by race range from $8-50 \%$ across the various patients populations that have been studied. The reasons for this persistent racial disparity in CRC survival remain poorly understood. However, results from the present study suggest that the effect of 'race' on advanced stage disease survival is not uniform across the population. Factors such as patient's age at diagnosis, gender, type of CRC, and date of diagnosis (possibly reflecting differences in treatment utilization) all influenced the relationship between race and survival.

On average, AA are known to be diagnosed with CRC at an earlier age $(11,12,49)$, but to our knowledge there are no previous reports of racial differences in survival by age. A large body of evidence documents that CRCs in younger persons are more likely to have familial hereditary CRC and, independently of family history, are likely to have more advanced and aggressive tumors $(41,42$ 2008, 43, 44). Younger patients also appear to have more genome complexity (assessed as copy number aberrations), and higher P53 expression or mutation $(41,42,45)$. Within our data, younger AA and EA had different tumor profiles. AA had a higher prevalence of proximally located $(+13 \%)$, mucinous $(+4 \%)$ and lower grade $(+6 \%)$ $\mathrm{CRCs}$ at diagnosis. Whether this represents a unique phenotype associated with poorer survival will need further study. On the other hand, despite the presence of more aggressive 
disease, many studies have shown that younger persons (predominantly EA) have better survival from CRC [20, 23, 24, 27]. Another possibility is that poor clinical performance status coupled with an aggressive phenotype added to the worse outcomes among AA, but we could not assess this in our dataset. Finally, the role of hereditary colon cancer in younger AA and its impact on survival is largely unexplored. Two studies have reported a higher prevalence of MSI-H tumors in AA - a common phenotype in familial CRC-compared to historical controls and, in one, the tumors exhibited a different mutational pattern $[32,33]$.

Outcomes in our investigation differed by race and by sex. Similar to two recent studies [28, 29], we observed that younger EA women had better survival than younger EA men. In the present study, however, young AA women had worse survival than younger EA women did. In part, the racial difference by gender in the young may be influenced by the colonic location of the CRC. For example, among younger women, proximal colonic location was associated with poorer survival and younger AA women had a higher prevalence of proximal tumors compared to EA ( $44 \%$ vs. 33\%). For older women, there was no difference in prevalence of proximally located tumors by race (AA $48 \%$ vs. EA 50\%) and no racial difference in survival. Several studies have shown that estrogen protects against CRC [34-36], and it is possible that a subset of younger EA women have a different hormonal profile compared to younger AA woman. A similar pattern of poorer survival and aggressive phenotypes has been observed in younger AA breast cancers patients $[37,38]$ compared to EA. Among advanced stage CRC patients older than 50 years, a modest increase in risk among AA men compared to EA was seen with no difference among women.

Many studies, like ours, have found that clinicopathologic characteristics influence survival. Similar to previous investigations [39-41], we observed the prevalence of proximally based tumors is higher in AA. As in the present study, several previous studies have previously observed that proximal colonic location is associated with worse survival [16-19, 42]. Compared to distal tumors, proximal colonic tumors were observed to be associated with greater mortality among stage IV CRC patients (HR 1.22; 95\% CI 1.16-1.28)) [16]. However, proximal cancers are not uniform. A subset of proximal tumors is associated with favorable prognosis (i.e. MSI-H cancers). CRC is increasingly recognized as a heterogeneous disease, which can evolve through at least three distinct carcinogenic pathways [43-49]: (1) the chromosomal instability pathways (CIN), (2) The CpG methylator pathway-1 (CIMP-1) and (3) the CpG methylator pathway-2 (CIMP-2). The CIMP pathways predominate in the proximal colon and demonstrate $\mathrm{CpG}$ methylation. The CIMP-1 cancers tend to exhibit MSI-H (Microsatellite instability-high) status, BRAF mutations, and low levels of KRAS and p53 mutations and have good prognosis; CIMP-2 CRCs have a higher proportion of Microsatellite stability (MSS), more frequent KRAS mutations and poor prognosis. The CIN pathway tumors show distal predominance, APC, P53 mutations, MSS, and lack extensive $\mathrm{CpG}$ methylation and have average prognosis. Furthermore, a recent study has found that AA have a higher prevalence of KRAS mutations, and those with these mutations have poorer survival [50]. While personalized medicine will certainly focus effective treatment on a colon tumor's molecular vulnerabilities, it may be some time before this knowledge can fully explain the disparities we observed in the present study. Future 
studies that integrate detailed molecular genetic/epigenetic markers along with detailed treatment information may be of particular value.

The persistence of racial differences in survival within the same stage suggests that treatment differences may be at least part of the explanation for differences in survival [3, 51]. Historically, many [3, 11, 51, 52], but not all, studies [14] have found that AA patients are less likely to receive standard recommended therapy, and refuse therapy at a higher rate. We observed no statistically significant differences in the initiation of chemotherpy by race, but did see striking improvements in survival among EA patients after the introduction of combination chemotherpy in 2000, and again with the introdution of bevacizumab to the standard of care in 2004. Treatment utilization is strongly influenced by access to highquality medical care. Medically underserved populations (including AA) are less likely to have equal access to the state-of-the art care, especially younger AA without health insurance [51]. As such, it may be that there is a racial disparity in the dissemination of the newer chemotherpies although a recent report did not observe this [15]. In health care settings where patients have equal access to care, the racial disparity in treatment utilization and outcomes is attenuated. In the Veterans Administration, for example, no significant differences by race were reported in rates of surgical resection, radiation therapy, or chemotherapy use. Although AA as compared to EA had modestly poorer survival [53], the disparity is less pronounced than commonly observed in population-based studies. However, treatment efficacy may also differ by race. Sanoff and colleagues [8] analyzed outcomes to combination chemotherapy within the context of a clinical trial and found that survival by race differed by type of treatment regimen. AA patients on FOLFOX or FOLFIRI had lower survival compared to EA, yet AA on IROX (i.e., no 5-FU) appeared to have better survival than EA. On the other hand, recently, Polite et al. [15] studied metastatic CRC patients who received bevacizumab within a community based setting and observed no difference in survival by race. In both of these studies, AA had significantly lower response rates. Because treatment regimen was not detailed in our data, it is difficult to assess the role of treatment use versus efficacy on survival by race. Furthermore, because we do not have information on the treatment medication administered to patients we cannot be certain that AA and EA actually received the same multi-drug regimens which, if different by race, could potentially contribute to some of the differences in survival.

Advantages of our study include a large, racially diverse population of patients with advanced stage colorectal cancer with careful characterization of demographic and pathologic characteristics and vital status. However, our study had a few limitations. First, we had no data on patient-level factors (such co-morbid conditions or lifestyle and behaviors) or detailed treatment regimen data, which confound or modify the association between race and CRC survival. Second, we did not have information on CRC specific deaths only on all cause mortality; however, because all of the patients had advanced stage CRC we expect that most of these people died a result of their disease. Finally, the setting in South Carolina may differ from other locales in ways that limit the generalizability of the study findings. For example, South Carolina has a high proportion of rural residents, which may introduce substantive differences in results compared to populations that are largely urban. In the future, it will be essential to see if our findings can be replicated in different populations, and future studies that integrate patient-level, clinical, molecular, and 
treatment-related data hold promise for advancing understanding of the racial disparity in CRC survival.

\section{Acknowledgments}

Funding for this research was from an American Cancer Society Institutional Research Grant awarded to the Hollings Cancer Center, Medical University of South Carolina (IRG-97-219-11); the National Cancer Institute via the Hollings Cancer Center NCI Cancer Center Support Grant (P30 CA138313), the Centers for Disease Control and Prevention cooperative agreement to the SC Dept of Health \& Environmental Control, Central Cancer Registry (DP12-1205) and a K07 Career Development Award to Dr. Wallace (K07CA151864-01A1).

\section{References}

1. Siegel R, Naishadham D, Jemal A. Cancer statistics, 2012. CA Cancer J Clin. 62:10-29. [PubMed: 22237781]

2. Freeman HP, Alshafie TA. Colorectal carcinoma in poor blacks. Cancer. 2002; 94:2327-32. [PubMed: 12015756]

3. Polite BN, Dignam JJ, Olopade OI. Colorectal cancer and race: understanding the differences in outcomes between African Americans and whites. Med Clin North Am. 2005; 89:771-93. [PubMed: 15925649]

4. Albano JD, Ward E, Jemal A, Anderson R, Cokkinides VE, Murray T, et al. Cancer mortality in the United States by education level and race. J Natl Cancer Inst. 2007; 99:1384-94. [PubMed: 17848670]

5. Robbins AS, Siegel RL, Jemal A. Racial disparities in stage-specific colorectal cancer mortality rates from 1985 to 2008. J Clin Oncol. 30:401-5. [PubMed: 22184373]

6. Soneji S, Iyer SS, Armstrong K, Asch DA. Racial disparities in stage-specific colorectal cancer mortality: 1960-2005. Am J Public Health. 100:1912-6. [PubMed: 20724684]

7. Kopetz S, Chang GJ, Overman MJ, Eng C, Sargent DJ, Larson DW, et al. Improved survival in metastatic colorectal cancer is associated with adoption of hepatic resection and improved chemotherapy. J Clin Oncol. 2009; 27:3677-83. [PubMed: 19470929]

8. Sanoff HK, Sargent DJ, Green EM, McLeod HL, Goldberg RM. Racial differences in advanced colorectal cancer outcomes and pharmacogenetics: a subgroup analysis of a large randomized clinical trial. J Clin Oncol. 2009; 27:4109-15. [PubMed: 19636001]

9. White A, Vernon SW, Franzini L, Du XL. Racial disparities in colorectal cancer survival: to what extent are racial disparities explained by differences in treatment, tumor characteristics, or hospital characteristics? Cancer. 116:4622-31. [PubMed: 20626015]

10. Demissie K, Oluwole OO, Balasubramanian BA, Osinubi OO, August D, Rhoads GG. Racial differences in the treatment of colorectal cancer: a comparison of surgical and radiation therapy between Whites and Blacks. Ann Epidemiol. 2004; 14:215-21. [PubMed: 15036226]

11. Baldwin LM, Dobie SA, Billingsley K, Cai Y, Wright GE, Dominitz JA, et al. Explaining blackwhite differences in receipt of recommended colon cancer treatment. J Natl Cancer Inst. 2005; 97:1211-20. [PubMed: 16106026]

12. Cooper GS, Yuan Z, Landefeld CS, Rimm AA. Surgery for colorectal cancer: Racerelated differences in rates and survival among Medicare beneficiaries. Am J Public Health. 1996; 86:582-6. [PubMed: 8604797]

13. Cook AD, Single R, McCahill LE. Surgical resection of primary tumors in patients who present with stage IV colorectal cancer: an analysis of surveillance, epidemiology, and end results data, 1988 to 2000. Ann Surg Oncol. 2005; 12:637-45. [PubMed: 15965730]

14. Le H, Ziogas A, Lipkin SM, Zell JA. Effects of socioeconomic status and treatment disparities in colorectal cancer survival. Cancer Epidemiol Biomarkers Prev. 2008; 17:1950-62. [PubMed: 18708384]

15. Polite BN, Sing A, Sargent DJ, Grothey A, Berlin J, Kozloff M, et al. Exploring racial differences in outcome and treatment for metastatic colorectal cancer: results from a large prospective observational cohort study (BRiTE). Cancer. 118:1083-90. [PubMed: 21800287] 
16. Meguid RA, Slidell MB, Wolfgang CL, Chang DC, Ahuja N. Is there a difference in survival between right- versus left-sided colon cancers? Ann Surg Oncol. 2008; 15:2388-94. [PubMed: 18622647]

17. Gatta G, Capocaccia R, De Angelis R, Stiller C, Coebergh JW, Group EW. Cancer survival in European adolescents and young adults. Eur J Cancer. 2003; 39:2600-10. [PubMed: 14642922]

18. O'Connell JB, Maggard MA, Ko CY. Colon cancer survival rates with the new American Joint Committee on Cancer sixth edition staging. J Natl Cancer Inst. 2004; 96:1420-5. [PubMed: 15467030]

19. Wray CM, Ziogas A, Hinojosa MW, Le H, Stamos MJ, Zell JA. Tumor subsite location within the colon is prognostic for survival after colon cancer diagnosis. Dis Colon Rectum. 2009; 52:135966. [PubMed: 19617745]

20. O'Connell JB, Maggard MA, Liu JH, Etzioni DA, Livingston EH, Ko CY. Do young colon cancer patients have worse outcomes? World J Surg. 2004; 28:558-62. [PubMed: 15366745]

21. Yantiss RK, Goodarzi M, Zhou XK, Rennert H, Pirog EC, Banner BF, et al. Clinical, pathologic, and molecular features of early-onset colorectal carcinoma. Am J Surg Pathol. 2009; 33:572-82. [PubMed: 19047896]

22. Wallace, K.; Seabrook, M.; Stinson, M.; Gilchrist, G.; Wortham, D.; Davis, A., et al. Results from a Colonoscopy Based Screening Program for the uninsured: a Pilot Study from South Carolina. ACG; San Antonio, TX: 2010. p. P829

23. Derwinger K, Kodeda K, Gerjy R. Age aspects of demography, pathology and survival assessment in colorectal cancer. Anticancer Res. 30:5227-31. [PubMed: 21187518]

24. Wang L, Hollenbeak CS, Stewart DB. Node yield and node involvement in young colon cancer patients: is there a difference in cancer survival based on age? J Gastrointest Surg. 14:1355-61. [PubMed: 20585992]

25. Perea J, Alvaro E, Rodriguez Y, Gravalos C, Sanchez-Tome E, Rivera B, et al. Approach to earlyonset colorectal cancer: clinicopathological, familial, molecular and immunohistochemical characteristics. World J Gastroenterol. 16:3697-703. [PubMed: 20677343]

26. Dozois EJ, Boardman LA, Suwanthanma W, Limburg PJ, Cima RR, Bakken JL, et al. Young-onset colorectal cancer in patients with no known genetic predisposition: can we increase early recognition and improve outcome? Medicine (Baltimore). 2008; 87:259-63. [PubMed: 18794708]

27. Hemminki K, Santi I, Weires M, Thomsen H, Sundquist J, Bermejo JL. Tumor location and patient characteristics of colon and rectal adenocarcinomas in relation to survival and TNM classes. BMC Cancer. 10:688. [PubMed: 21176147]

28. Hendifar A, Yang D, Lenz F, Lurje G, Pohl A, Lenz C, et al. Gender disparities in metastatic colorectal cancer survival. Clin Cancer Res. 2009; 15:6391-7. [PubMed: 19789331]

29. Koo JH, Leong RW. Sex differences in epidemiological, clinical and pathological characteristics of colorectal cancer. J Gastroenterol Hepatol. 25:33-42. [PubMed: 19874446]

30. Qaseem A, Denberg TD, Hopkins RH Jr. Humphrey LL, Levine J, Sweet DE, et al. Screening for colorectal cancer: a guidance statement from the American College of Physicians. Ann Intern Med. 156:378-86. [PubMed: 22393133]

31. Rex DK, Johnson DA, Anderson JC, Schoenfeld PS, Burke CA, Inadomi JM, et al. American College of Gastroenterology guidelines for colorectal cancer screening 2009 [corrected]. Am J Gastroenterol. 2009; 104:739-50. [PubMed: 19240699]

32. Ashktorab H, Smoot DT, Farzanmehr H, Fidelia-Lambert M, Momen B, Hylind L, et al. Clinicopathological features and microsatellite instability (MSI) in colorectal cancers from African Americans. Int J Cancer. 2005; 116:914-9. [PubMed: 15856472]

33. Kumar K, Brim H, Giardiello F, Smoot DT, Nouraie M, Lee EL, et al. Distinct BRAF (V600E) and KRAS mutations in high microsatellite instability sporadic colorectal cancer in African Americans. Clin Cancer Res. 2009; 15:1155-61. [PubMed: 19190129]

34. Anderson GL, Limacher M, Assaf AR, Bassford T, Beresford SA, Black H, et al. Effects of conjugated equine estrogen in postmenopausal women with hysterectomy: the Women's Health Initiative randomized controlled trial. Jama. 2004; 291:1701-12. [PubMed: 15082697] 
35. Newcomb PA, Longnecker MP, Storer BE, Mittendorf R, Baron J, Clapp RW, et al. Long-term hormone replacement therapy and risk of breast cancer in postmenopausal women. Am J Epidemiol. 1995; 142:788-95. [PubMed: 7572954]

36. Mandelson MT, Miglioretti D, Newcomb PA, Harrison R, Potter JD. Hormone replacement therapy in relation to survival in women diagnosed with colon cancer. Cancer Causes Control. 2003; 14:979-84. [PubMed: 14750537]

37. Amend K, Hicks D, Ambrosone CB. Breast cancer in African-American women: differences in tumor biology from European-American women. Cancer Res. 2006; 66:8327-30. [PubMed: 16951137]

38. Carey LA, Perou CM, Livasy CA, Dressler LG, Cowan D, Conway K, et al. Race, breast cancer subtypes, and survival in the Carolina Breast Cancer Study. JAMA. 2006; 295:2492-502. [PubMed: 16757721]

39. Shavers VL. Racial/ethnic variation in the anatomic subsite location of in situ and invasive cancers of the colon. J Natl Med Assoc. 2007; 99:733-48. [PubMed: 17668639]

40. Thornton JG, Morris AM, Thornton JD, Flowers CR, McCashland TM. Racial variation in colorectal polyp and tumor location. J Natl Med Assoc. 2007; 99:723-8. [PubMed: 17668638]

41. Ashktorab H, Nouraie M, Hosseinkhah F, Lee E, Rotimi C, Smoot D. A 50-year review of colorectal cancer in African Americans: implications for prevention and treatment. Dig Dis Sci. 2009; 54:1985-90. [PubMed: 19554449]

42. Kalady MF, de Campos-Lobato LF, Stocchi L, Geisler DP, Dietz D, Lavery IC, et al. Predictive Factors of Pathologic Complete Response After Neoadjuvant Chemoradiation for Rectal Cancer. Ann Surg. 2009

43. Shen L, Toyota M, Kondo Y, Lin E, Zhang L, Guo Y, et al. Integrated genetic and epigenetic analysis identifies three different subclasses of colon cancer. Proc Natl Acad Sci U S A. 2007; 104:18654-9. [PubMed: 18003927]

44. Suehiro Y, Wong CW, Chirieac LR, Kondo Y, Shen L, Webb CR, et al. Epigeneticgenetic interactions in the APC/WNT, RAS/RAF, and P53 pathways in colorectal carcinoma. Clin Cancer Res. 2008; 14:2560-9. [PubMed: 18451217]

45. Jass JR. Classification of colorectal cancer based on correlation of clinical, morphological and molecular features. Histopathology. 2007; 50:113-30. [PubMed: 17204026]

46. Ogino S, Nosho K, Kirkner GJ, Kawasaki T, Meyerhardt JA, Loda M, et al. CpG island methylator phenotype, microsatellite instability, BRAF mutation and clinical outcome in colon cancer. Gut. 2008

47. Kaneda A, Yagi K. Two groups of DNA methylation markers to classify colorectal cancer into three epigenotypes. Cancer Sci. 102:18-24. [PubMed: 21159060]

48. Issa JP. CpG island methylator phenotype in cancer. Nat Rev Cancer. 2004; 4:988-93. [PubMed: 15573120]

49. Issa JP. Colon cancer: it's CIN or CIMP. Clin Cancer Res. 2008; 14:5939-40. [PubMed: 18829469]

50. Sylvester BE, Huo D, Khramtsov A, Zhang J, Smalling RV, Olugbile S, et al. Molecular analysis of colorectal tumors within a diverse patient cohort at a single institution. Clin Cancer Res. 18:350-9. [PubMed: 22114137]

51. Mitchell EP. Prognostic impact of race and ethnicity in the treatment of colorectal cancer. Med Clin North Am. 2005; 89:1045-57. 1054. [PubMed: 16129111]

52. Schrag D, Gelfand SE, Bach PB, Guillem J, Minsky BD, Begg CB. Who gets adjuvant treatment for stage II and III rectal cancer? Insight from surveillance, epidemiology, and end results-Medicare. J Clin Oncol. 2001; 19:3712-8. [PubMed: 11533092]

53. Dominitz JA, Samsa GP, Landsman P, Provenzale D. Race, treatment, and survival among colorectal carcinoma patients in an equal-access medical system. Cancer. 1998; 82:2312-20. [PubMed: 9635522] 

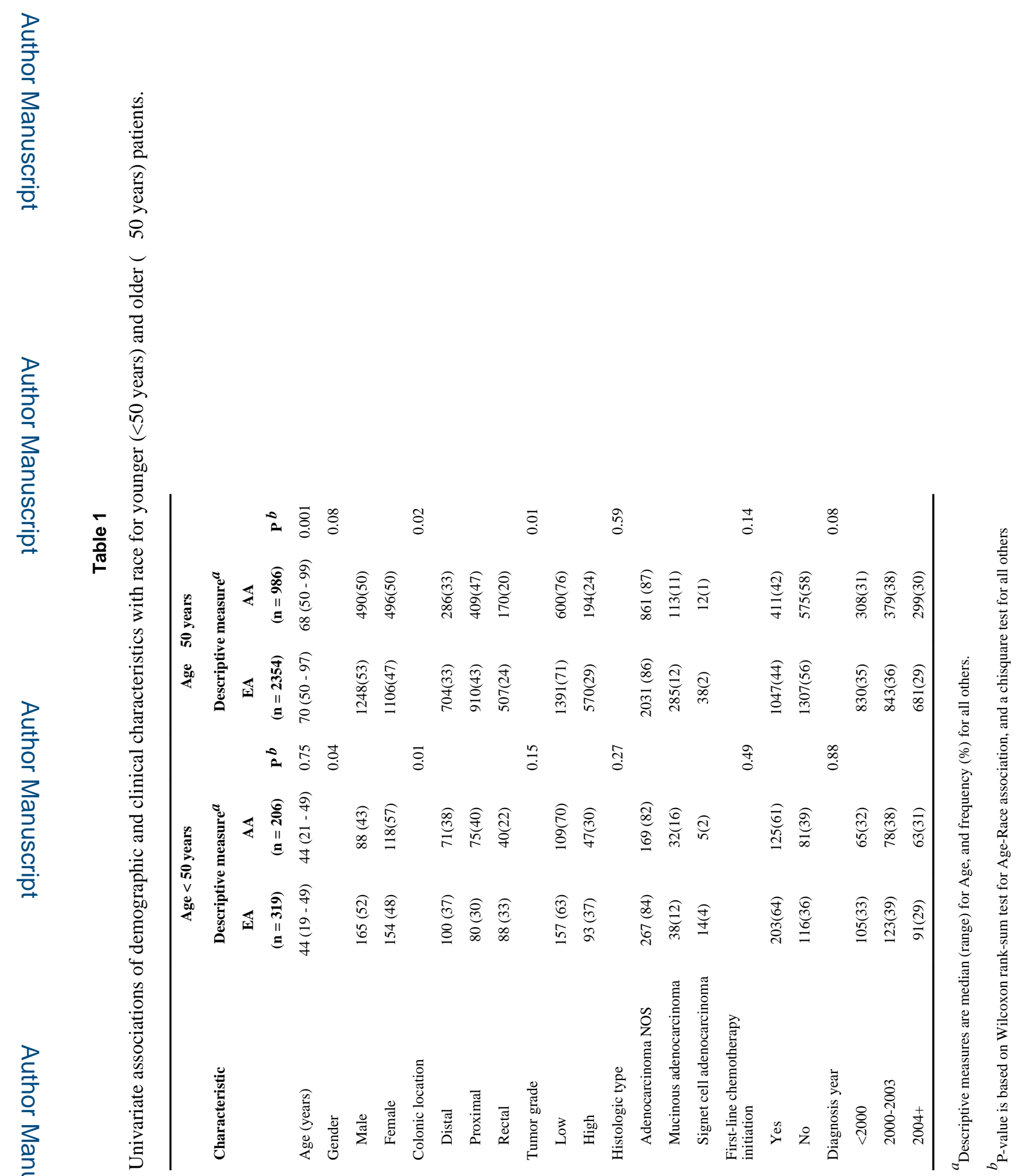


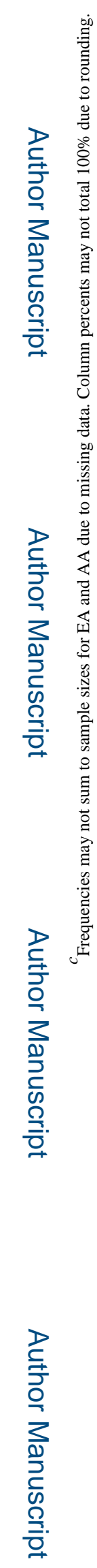




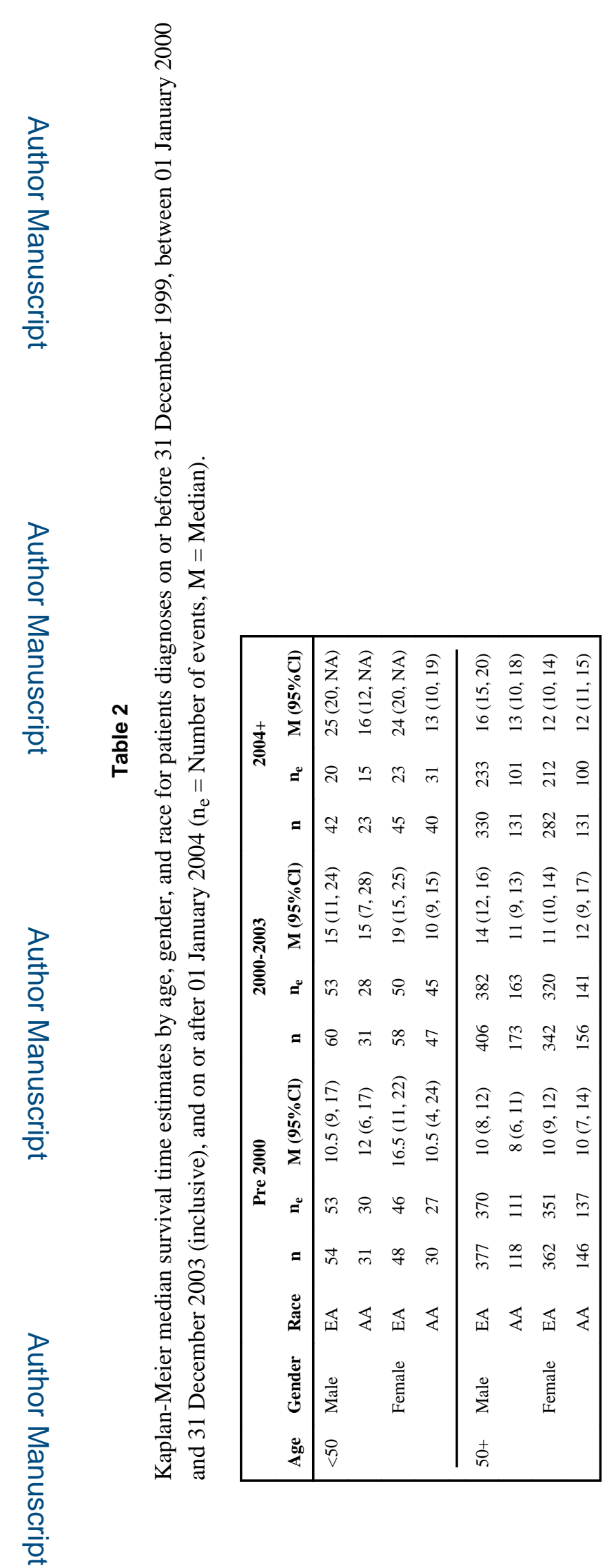


Wallace et al.

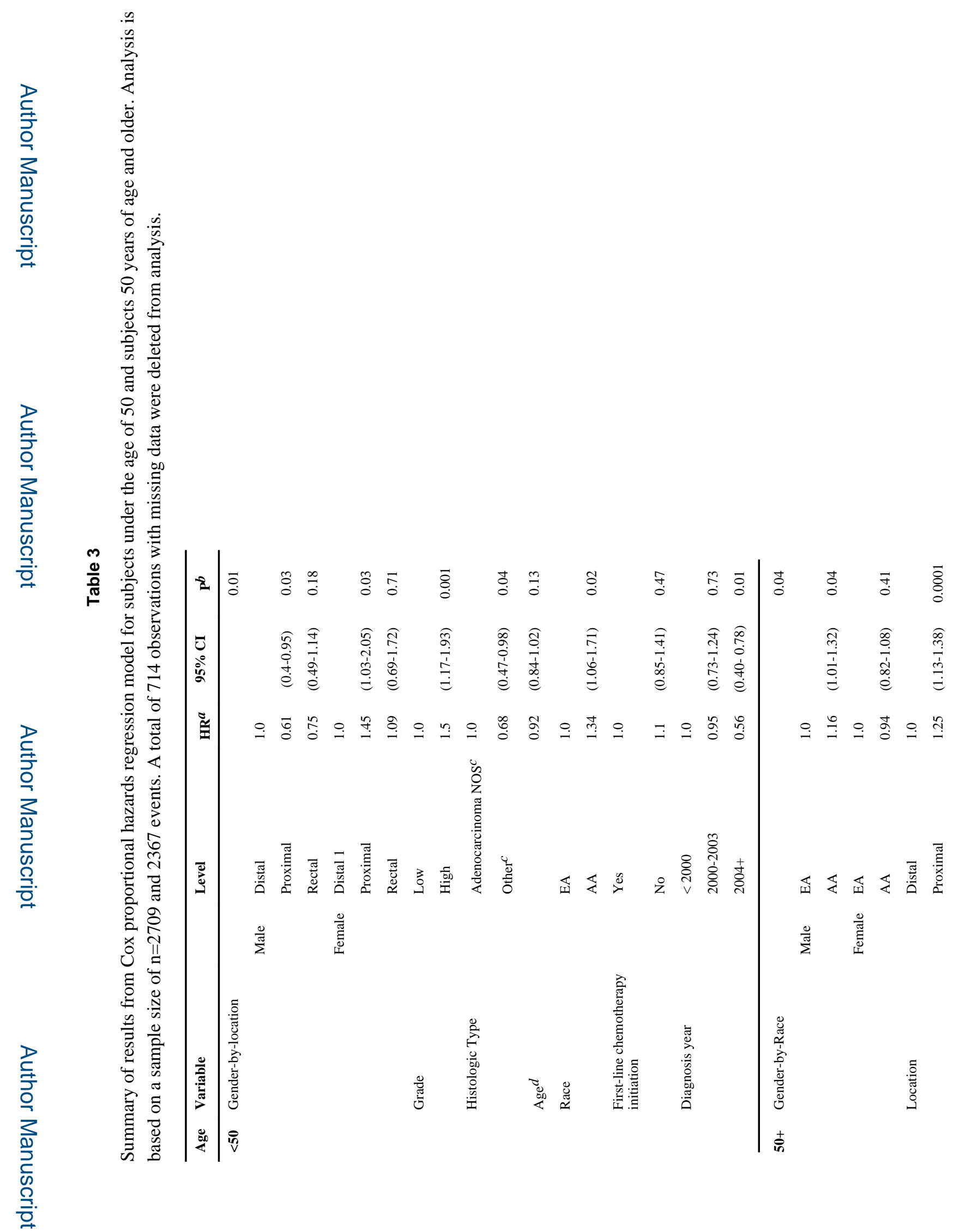

Cancer Causes Control. Author manuscript; available in PMC 2014 March 01. 


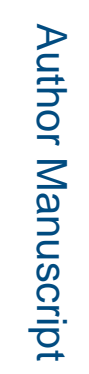

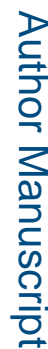

롬

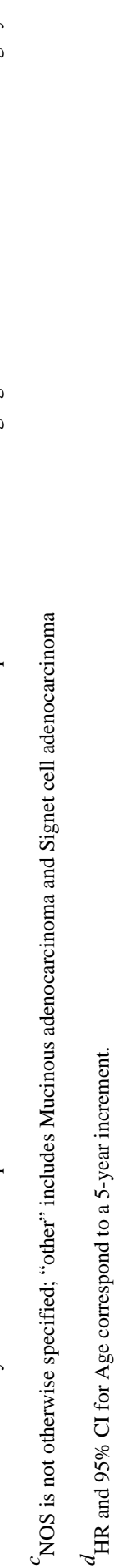

Cancer Causes Control. Author manuscript; available in PMC 2014 March 01. 